\title{
Consequences of Priming: Judgment and Behavior
}

\author{
Paul M. Herr \\ Graduate School of Business, \\ Indiana University
}

\begin{abstract}
Two experiments investigated the judgmental and behavioral consequences of priming a social category. In the first experiment, assimilation and contrast effects of judgment of a target person's hostility obtained following priming with exemplars of, respectively, moderate and extreme levels of the category hostility. The second experiment replicated these findings and, in addition, demonstrated that subjects then behaved consistently with their evaluations of the target person in a social interaction. The results are discussed in terms of the social interaction literature, with category accessibility serving as a means of creating an expectancy for the target's behavior.
\end{abstract}

A considerable amount of research evidence supports the notion that the expectations held by an individual regarding the behavior of a target person determine, to a great extent, the content of the social interaction between the two individuals. In game settings, subjects who thought that their partner disliked them (Jones \& Panitch, 1971) or who were led to believe that their partner was a generally hostile person (Snyder \& Swann, 1978) behaved significantly more competitively toward that person than when such an expectancy was not present. Kelley and Stahelski (1970) found that subjects who expected their partners to be competitive behaved in a more competitive manner as well. In each case, subjects' expectancies of another individual were manipulated and the subject holding that expectancy came to act differently toward the target person.

The effects of expectancies on social interactions are not confined to game situations. Word, Zanna, and Cooper (1974) found that, in the context of an interracial interview, black "job applicants" received shorter interview times, greater physical distancing, and higher rates of speech errors from white interviewers than did white applicants. Snyder, Tanke, and Berscheid (1977) demonstrated that men's beliefs that women who were physically attractive would be warmer and more sociable than physically unattractive women resulted in different behavior to a female target in a telephone conversation.

All of these studies provide evidence that individuals' expectations indeed affect the nature of a behavioral interaction. Whether the expectancy was formed in the lab by an experimenter or held by the subject before, the social interaction with

This article is based on a doctoral dissertation conducted at Indiana University and supported by an Indiana University doctoral dissertation grant.

I would like to thank the members of the dissertation committee, Russel H. Fazio, Steven J. Sherman, Jerome M. Chertkoff, and Donald A. Wood. I would like to repeat my expressions of thanks to Russell $\mathrm{H}$. Fazio and Martha C. Powell for comments on an earlier draft of this article.

Correspondence concerning this article should be addressed to Paul M. Herr, Graduate School of Business, Indiana University, $801 \mathrm{~W}$. Michigan, Indianapolis, Indiana 46223. a target person was profoundly influenced by the subject's belief of how the target would behave.

Because expectancies are obviously so important, it becomes equally important to understand what determines the individual's immediate perceptions. It is possible that even when individuals encounter strangers for whom they have no distinct preconceptions, the same consequences as reported in the social interaction literature may occur. Just as attitudes (or associations between objects and evaluations of those objects) and stereotypes can shape the immediate perceptions of familiar persons or objects, the recent activation or use of different, more basic cognitive categories (e.g., general knowledge structures) may influence our immediate perceptions of persons with whom we are unfamiliar. The difference between this approach and the older social interaction literature lies in what categories are activated before the social interaction. In the literature, the category is generally an attitude or a stereotype, or an expectancy is directly manipulated by experimenters' instructions. In this article, I argue that a more general category, when activated prior to a judgment on the part of the perceiver, can have the same behavioral consequences. This more general category can be thought of as an object-free description or set of rules for classifying an object or person, or it simply can be thought of as a set of exemplars of that category. The purpose of the present research is to examine the possibility of this more subtle process of social interaction; a process that is initiated not by experimental instructions for the participants to expect a certain kind of individual, nor by prior beliefs that the individual is a certain kind of person, but rather by the recent activation from memory of a general category, which itself may then determine the expectancy of how another person will behave.

A process whereby this may occur is suggested by recent findings in the social cognition literature. Subjects primed with exemplars of a particular category are more likely to use that category in evaluating a subsequently presented category-relevant stimulus (Higgins, Rholes, \& Jones, 1977; Srull \& Wyer, 1978). These results have been conceptualized in a number of ways. Higgins and King (1981) and Higgins, Bargh, and Lombardi (1985) have suggested an energy cell process in which the energy or action potential of a category is increased whenever the cell involved is activated (as by recent or frequent exposure 
to exemplars of the category). Any new stimulus is encoded by the relevant cell with the greatest action potential. Hence, priming a category increases the likelihood that it will be applied to new incoming information, so long as the category is applicable to the stimulus. The stimulus will then be categorized as an instance of that category.

An alternative conceptualization of priming effects is Wyer and Srull's (1980a, 1980b) storage bin model. In this model, a recently primed category is placed on top of a storage bin. When new information is processed, the category at the top of the bin is accessed first for encoding that new information, provided that the category is relevant and applicable. Both of these models hold that an activated category serves as a basis for interpreting incoming information and, provided the category is relevant and applicable to the incoming information, that information will be judged as an instance of or consistent with the category.

It has been demonstrated further however, that priming can also result in judgments inconsistent with, and opposite in nature to, the primed category (Herr, Sherman, \& Fazio, 1983; Martin, 1986). In the Herr et al. study, subjects were primed with exemplars of varying levels of animal size prior to making judgments about ambiguous-sized animals in an ostensibly unrelated experiment. The authors demonstrated that priming with exemplars of extreme categories prior to judgment of an unreal (ambiguous) animal resulted in these contrast effects. That is, subjects primed with exemplars of the category $e x$ tremely small animals later judged unreal animals to be larger than did subjects primed with exemplars of the category $e x$ tremely large animals. The authors also demonstrated the more commonly found assimilation effects when moderately extreme categories were primed prior to judgment of unreal animals. These findings were replicated in a second study in which animal ferocity was primed prior to judgment of the ferocity of unreal animals.

These first demonstrations of priming-induced assimilation and contrast effects in judgments of the same target stimuli suggest a mechanism for priming effects on judgment that extends the aforementioned models to include both memory and judgment effects. Specifically, when subjects are asked to evaluate a target stimulus along some dimension, a memory search for appropriate category membership is conducted. As these models both posit, priming serves to increase the likelihood of the primed category being accessed first in this memory search.

Whether the generally reported assimilation effects or contrast effects will occur seems to be in part a function of the degree of overlap between the features of the primed category and the stimulus to be evaluated. To the extent that a comparison of features of the activated category and the target stimulus results in matching or overlap, a judgment of category membership should occur. In the case of a moderate category and an ambiguous target, the potential for feature matching is relatively high, and the target should in fact be judged as an instance of that category. Having categorized the target, the subject must still evaluate it along the relevant dimension (e.g., "How large is it?"). In this case, the judgment will be made by selecting the average level of category members along the chosen dimension. In the case of a moderately extreme category and an ambiguous target (which heretofore had been the only stimuli involved in priming work), assimilation effects should be likely (and in fact were the only reported findings; Bargh \& Pietromonaco, 1982; Higgins et al., 1977; Srull \& Wyer, 1978, 1979).

The mechanism whereby contrast effects occur is essentially the same in nature. The features of the primed category are compared with the features of the target, and a judgment with respect to category membership is made. When extreme categories are primed, there should be very little overlap or match between features of that category and the target. Given the lack of feature matching, the ambiguous target should not be categorized within the primed category. In the absence of categorization, the extreme category nonetheless has an effect on the judgment of the target along the evaluation dimension. Specifically, the priming exemplars serve as anchors or standards of comparison for the target. Ostrom and Upshaw (1968) and Upshaw (1962) have suggested that when subjects are asked to evaluate a stimulus along some dimension, they typically align the given scale with the range of stimuli they expect to evaluate or with the range considered at the time of judgment. If the contextual stimuli (produced either by actively rating other stimuli or through a memory search biased through prior passive priming) are extreme (i.e., toward one end of the rating scale), the judge will position the response scale toward that end of the continuum. Consequently, judges considering generally large values will make smaller ratings of a target than will judges whose range has been broadened at the small end owing to consideration of contextual stimuli that are small.

This discussion suggests that the predominance of assimilation effects when social categories are primed may be due, in part, to priming with exemplars of only moderately extreme categories. No study, except Herr et al. (1983), has specifically attempted to manipulate extremity. This may be due, in part, to the priming procedures and exemplars used. For instance, Srull and Wyer $(1978,1979)$ used a sentence-completion task to activate social categories. It simply may be difficult to activate extreme social categories in this manner.

Once a category has been activated, it may then affect an expectation in the previously described manner. To the extent that the activated category results in a judgment of category inclusion of the stimulus object, that judgment should increase the expectancy of that object's inclusion in the category at some future point. Individuals may be unaware (as in the priming examples; see Higgins et al., 1977, and Bargh \& Pietromonaco, 1982) or aware (as in some of the examples from the social interaction literature) of their expectancies. In the latter case, it has already been shown that such expectancies have had marked effects on both judgments and later behavior.

Given the findings of Herr et al. (1983) that, in addition to producing categorization effects, an activated category can also set a context for judgments of size and ferocity of animals, it seems reasonable to expect this effect following priming with exemplars of a social category. That is, the activated category should set a context for judgments in a social domain, and following judgments, behavior should be consistent with the judgment. In Experiment 1 I will attempt to demonstrate the generalizability of the context effects of priming to a social domain using famous persons as exemplars of social categories. In Experiment 2 , an attempt will be made to demonstrate the behav- 
Table 1

Mean Ratings of Exemplars of Hostility

\begin{tabular}{lcccc}
\hline & \multicolumn{3}{c}{ Categories } \\
\cline { 2 - 5 } Item & Extremely nonhostile & Moderately nonhostile & Moderately hostile & Extremely hostile \\
\hline \multirow{2}{*}{ Exemplars } & Peter Pan & Daniel Boone & Menachem Begin & Dracula \\
& Pope John Paul & Robin Hood & Alice Cooper & Ayatollah Khomeini \\
& Shirley Temple & Billie Jean King & Joe Frazier & Adolph Hitler \\
$M$ & Santa Claus & Henry Kissinger & Bobby Knight & Charles Manson \\
$S D$ & 1.04 & 4.29 & 6.77 & \\
& 1.49 & 2.07 & 2.28 & 1.35 \\
\hline
\end{tabular}

Note. Higher scores indicate greater perceived hostility on an 11-point scale.

ioral consequences of category activation on a social interaction between a primed subject and a naive target person.

The first experiment was conducted to examine the judgmental consequences of priming the social category hostility. It was expected that subjects primed with exemplars of extreme hostility or extreme nonhostility would display contrast effects in their judgment of an ambiguously described target person's hostility, whereas those subjects primed with exemplars of moderate hostility or moderate nonhostility would display assimilation to those categories. That is, subjects primed with exemplars of moderate hostility or extreme nonhostility should evaluate the target as being more hostile than should subjects who have been primed with exemplars of extreme hostility or moderate nonhostility.

\section{Experiment 1}

\section{Method}

Subjects. The subjects were 160 Indiana University female undergraduates. They were run in groups of up to 20 , and they participated in order to fulfill part of their introductory psychology research requirement.

Stimuli. Pretesting revealed four groups of famous persons perceived as personifying different levels of hostility. Four names in each group were selected on the basis of the responses of 65 introductory psychology students, who also participated to fulfill part of a course requirement. Each subject responded to 249 names and evaluated each name on an 11-point scale that ranged from not at all hostile to extremely hostile. Table 1 presents the four groups of names (ranging from $e x$ treme nonhostility to extreme hostility) that were used as primes in the experiments.

Procedure. In order to detect the judgmental effects of priming, subjects were exposed to one of the four lists of famous persons' names and then were asked to read a short, ambiguous description of a target person and evaluate his or her hostility.

The priming took place in the context of a study examining personality and problem-solving abilities. Each subject received a booklet that consisted of filler puzzle pages alternating with pages containing questions designed to assess individual differences in accessibility of the category hostility. (After Higgins, King, and Mavin, 1982, listing of the trait hostility, or a close synonym, would reflect accessibility of the category.) These questions represented an exploratory aspect of the study and were presented to determine the effect of individual differences in accessibility of the category hostility on later judgments as well as to determine any possible interaction with priming. As this was a peripheral aspect of the study, it will not be further discussed here. The final puzzle in each booklet represented the priming manipulation. The puzzle was a $20 \times 20$ matrix of letters with one of the four groups of names in Table 1 embedded therein. Subjects were instructed to circle each of the four names in the matrix. A list of the names in the puzzle was provided to ensure that all names were found.

In order to detect whether trait listing activated categories that would interact with the priming manipulation, half of the subjects were given booklets consisting solely of the four filler puzzles and the priming puzzle. Trait listing was then treated as a separate factor in the design.

Upon completion of the booklet, a second experimenter gave each subject a second booklet to complete. On the first page of the booklet was a paragraph identifying the experiment as a study investigating reading comprehension. On the second page was an ambiguous description of a target person's behavior. The description consisted of an account of "Donald," whose behavior could be categorized as either hostile or not hostile. The description was a modification of the one used by Srull and Wyer (1979). ${ }^{1}$ After reading the description, data on the dependent measure were collected by asking subjects to evaluate Donald's hostility along the 11-point scale described earlier.

In order to examine possible effects of priming on evaluations related to the category primed, several supplementary questions about Donald were asked, including the assessment of Donald's friendliness, the degree to which he seemed like someone the subject would like to know, and other evaluations potentially related to hostility. These supplementary questions all appeared on following pages, separated from the primary dependent measure regarding hostility.

\section{Results}

Separate 2 (extremity of prime: moderate or extreme) $\times 2$ (category primed: hostile or nonhostile) $\times 2$ (trait listing vs. notrait listing) analyses of variance (ANOVAs) were conducted on each of the questions answered following priming and on composites of questions that will be described later. No main effect for listing versus not listing traits obtained, and no three-way interaction even approached significance. There were occasional two-way interactions of extremity of prime and trait listing, which always took the form of more extreme ratings occurring following priming with moderate exemplars when traits

\footnotetext{
${ }^{1}$ To ensure that the description was in fact neutral, it was presented to 48 introductory psychology students who were asked to evaluate the hostility of Donald along an 11-point scale with endpoints labeled not at all hostile and extremely hostile. The mean rating was approximately at the midpoint of the same scale subjects used to select the exemplars $\left(M=5.27, S^{2}=2.33\right)$.
} 
Table 2

Dependent Measures for Experiment 1

\begin{tabular}{lcccc}
\hline & \multicolumn{4}{c}{ Prime } \\
\cline { 2 - 5 } & \multicolumn{2}{c}{ Moderate } & Extreme \\
\cline { 2 - 5 } Measure & Nonhostile & Hostile & Nonhostile & Hostile \\
\cline { 2 - 5 } & & & & \\
$\begin{array}{l}\text { Mean evaluation of } \\
\quad \text { hostility }\end{array}$ & $5.69_{\mathrm{a}}$ & $6.82_{\mathrm{b}}$ & $6.71_{\mathrm{b}}$ & $5.17_{\mathrm{a}}$ \\
$\begin{array}{l}\text { Supplementary ratings } \\
\text { Friendliness }\end{array}$ & $4.13_{\mathrm{ab}}$ & $4.00_{\mathrm{ab}}$ & $3.76_{\mathrm{a}}$ & $4.67_{\mathrm{b}}$ \\
$\quad$ Kindness & $3.87_{\mathrm{ab}}$ & $3.46_{\mathrm{ab}}$ & $2.95_{\mathrm{a}}$ & $4.20_{\mathrm{b}}$ \\
Composite ratings & & & & \\
Negative & $44.27_{\mathrm{a}} \mathrm{a}$ & $47.51_{\mathrm{a}}{ }^{\mathrm{a}}$ & $47.05_{\mathrm{a}}{ }^{\mathrm{a}}$ & $44.00_{\mathrm{a}}{ }^{\mathrm{a}}$ \\
Positive & $12.07_{\mathrm{a}}$ & $12.03_{\mathrm{a}}$ & $10.51_{\mathrm{a}}$ & $14.80_{\mathrm{b}}$ \\
Total & $32.25_{\mathrm{ab}}$ & $35.49_{\mathrm{ab}}$ & $36.54_{\mathrm{a}}$ & $29.20_{\mathrm{b}}$ \\
\hline
\end{tabular}

Note. Higher scores indicate that more of the perceived characteristic is attributed to the target. Cell means in the same row not sharing a common subscript differ beyond $p=.05$ by relevant test of simple effects.

${ }^{\mathrm{a}}$ Means differ at .10 by relevant test of simple effects.

were listed than when traits were not listed. It is not clear why the interaction took this form, but in any event, it was never sufficient to produce a three-way interaction. That is, the same general pattern of priming effects obtained irrespective of listing or not listing traits.

Of most importance is the significant interaction between extremity of priming exemplar and category primed in the first question. Just as predicted, subjects exposed to exemplars of extreme hostility or moderate nonhostility evaluated Donald as being significantly less hostile than did subjects exposed to exemplars of extreme nonhostility or moderate hostility, $F(1$, $151)=17.67, p<.001$. The means for this interaction are presented in Table 2.

Analyses of simple effects showed, as predicted, both assimilation and contrast effects. Subjects primed with exemplars of extreme nonhostility rated Donald as being significantly more hostile than did subjects primed with exemplars of extreme hostility, $t(151)=3.50, p<.01$, whereas subjects primed with exemplars of moderate nonhostility evaluated Donald as being significantly less hostile than did subjects primed with exemplars of moderate hostility, $t(151)=2.52, p<.05$. The only other effect to attain significance in the analysis was the extremity by trait versus no-trait listing interaction, $F(1,151)=4.99$, $p<.03$. These effects were not great enough, however, to produce a three-way interaction. The expected contrast effect after priming with extreme exemplars and assimilation effect after priming with moderate exemplars occurred whether traits were listed or not.

The remaining items on the questionnaire were designed to tap categories related to hostility. Analyses of variance were conducted on the rest of the measures as well as on composites of the measures. The individual items that attained significance were the questions assessing Donald's friendliness and kindness, each of which resulted in the an interaction between extremity and category primed, $F(1,151)=3.30, p<.07$, and $F(1,151)=8.79, p<.003$, respectively, and no main effects. Subjects exposed to exemplars of extreme hostility or moderate nonhostility perceived Donald as being both more friendly and more kind than did persons primed with exemplars of extreme nonhostility or moderate hostility. Tests for simple effects for this interaction and all subsequent analyses are presented along with the means in Table 2.

The items on the questionnaire that subjects completed following priming can be divided into two general classes: those dealing with generally positive characteristics of Donald (e.g., friendliness, kindness, how much the subject would like to know and work with Donald) and those dealing with generally negative characteristics (e.g., hostility, belligerence, bitterness). In order to arrive at an overall measure of impressions of Donald, reliabilities of the total scale and the two subscales (the positive questions and the negative questions alone) were calculated, and analyses were conducted on the sum of the items in each scale. Cronbach's alpha for the total scale was calculated to be .80 (for the negative attribute scale, $\alpha=.77$, and for the positive scale, $\alpha=.78$ ). These alphas were large enough to warrant analyses of the total scale taken as a unit (accomplished by positively weighting all positive questions and negatively weighting all negative questions and then summing all responses for each subject) and the subscales taken alone. For the total scale, these sums were then subjected to a 2 (category primed: hostile vs. nonhostile) $\times 2$ (extremity of priming exemplar: moderate vs. extreme) $\times 2$ (traits listed vs. no traits listed) ANOVA. As expected, the extremity of category primed by category primed interaction attained statistical significance, $F(1,151)=7.17$, $p<.008$, which indicated that subjects exposed to exemplars of extreme hostility or moderate nonhostility evaluated Donald in a more positive manner than did subjects exposed to exemplars of extreme nonhostility or moderate hostility. The same analysis conducted on the negative-item scale also revealed an interaction between extremity of category primed and category primed, $F(1,152)=5.55, p=.02$, in which subjects exposed to exemplars of extreme hostility or moderate nonhostility rated Donald less negatively than did subjects exposed to exemplars of moderate hostility or extreme nonhostility. When only positive items were considered, the ANOVA again revealed a signifcant interaction between extremity and category primed, $F(1$, $152)=4.39, p<.05$, in which subjects exposed to exemplars of extreme nonhostility or moderate hostility rated Donald less positively than did subjects exposed to exemplars of extreme hostility or moderate nonhostility. Simple effects analyses are presented in Table 2.

In sum, analyses of the supplementary measures revealed an interaction between category primed and the extremity of that category, with subjects exposed to exemplars of extreme hostility or moderate nonhostility rating Donald more favorably than did subjects exposed to exemplars of extreme nonhostility or moderate hostility. Although the interactions were in each case significant, analyses of the simple effects that make up the interactions revealed a general pattern of contrast effects when extreme categories were primed, and although the means were clearly in the predicted direction, at best only marginal assimilation effects obtained when moderate categories were primed. These contrast and assimilation effects seemed to be stronger when subjects were evaluating negative characteristics, yet contrast still occurred for positive judgments. Why these differences occurred is unclear.

Even though the priming exemplars were selected on the ba- 
sis of pretest subjects' evaluations of their hostility, it is possible that some other category (e.g., kindness or friendliness) was activated as well. The judgments of negative characteristics were entirely consistent with the notion that hostility was primed. Judgments of the supplementary positive characteristics, on the other hand, might lead one to believe that a category such as kindness or friendliness was activated and produced only contrast effects; that is, judgments as a negative function of the primes' hostility, independent of extremity.

Whatever category was primed, however, at least the tendency for both assimilation and contrast surfaced, as evidenced by crossover interactions for each rating. Contrast was simply much stronger in the supplementary positive ratings than was assimilation.

Perhaps the reason for this anomaly rests in the exemplars being less than perfect. Earlier priming studies used relatively unambiguous exemplars; either trait adjectives or behavioral examples. The present study used famous persons embodying defining characteristics of the category. Persons embodying extreme levels of nonhostility or hostility were also fairly unambiguous along those dimensions. Those characteristics were more or less central to their description. They were strongly identified with that characteristic. Persons identified as moderately hostile or nonhostile may be more strongly identified along some other dimension and only weakly with respect to hostility. Subjects were able to evaluate the exemplars' hostility on a scale, yet that might not have guaranteed that moderate hostility was central to the exemplar's definition and would be activated spontaneously upon observation of the exemplar. The moderate primes clearly did have some effect, however, as evidenced by the strong assimilation effects for hostility judgments and the crossover interactions for all judgments. That the effects were not stronger for the supplementary positive evaluations may be a simple consequence of their relative ambiguity and, hence, lack of potency as primes relative to the extreme exemplars.

\section{Discussion}

These results serve to replicate and extend the findings of Herr et al. (1983) with the use of social stimuli. That is, the results demonstrate both assimilation and contrast effects as consequences of priming categories relevant to social stimuli. Heretofore, priming-induced contrast effects had been demonstrated only following priming with exemplars of animal size and ferocity. Although it is important to demonstrate the existence of the effect, it is perhaps more important to show the reliability of the effect and its generalizability to more real-life situations. What has been shown here is that the effect obtains even for judgments of stimuli that are more complex and have potentially greater consequences.

\section{Experiment 2}

A second experiment was conducted to determine the effects of priming a social category on a behavioral interaction. It was expected that persons who were primed with exemplars of extreme hostility or moderate nonhostility would expect an unknown, ambiguously described target person to be less hostile than would subjects exposed to exemplars of extreme nonhostility or moderate hostility. That is, it was anticipated that the judgment effects demonstrated in the first experiment would be replicated. It was further expected that the primed persons' behavior would be mediated by these judgment effects; specifically, that they would behave consistently with their expectations. Following a judgment of the target person, a prisoners' dilemma game was played. Not only, it was hypothesized, should subjects exposed to exemplars of extreme hostility rate the target as less hostile than subjects exposed to exemplars of extreme nonhostility, they should also cooperate more (and hence compete less) with the target person they just evaluated. Subjects primed with exemplars of moderate nonhostility should also compete less and cooperate more with their partner (who they also should evaluate as being less hostile) than subjects primed with exemplars of moderate hostility. The procedure should also permit the detection of a self-fulfilling prophecy. Target persons in all conditions may reciprocate the behavior of the perceivers with whom they are paired and, hence, fulfill the perceivers' original expectation. The expectancy that was eventually confirmed was established not by experimenter's instruction, as in the social interaction literature, but rather as a consequence of either contrast away from or assimilation to that category activated by priming.

\section{Method}

Subjects. Subjects were 80 Indiana' University female undergraduates who participated to fulfill part of their introductory psychology research requirement.

Procedure. This experiment was conducted in the context of a study investigating first impressions. Subjects participated in pairs. In order to ensure that subjects never saw each other, they were instructed to come to separate rooms in the psychology building. At the appropriate time, a second experimenter brought the target person into the lab, making sure that the subjects never saw each other. Upon arrival in the lab, subjects were placed in separate booths and the experimenter explained to one subject (the perceiver), selected at random,

We are interested in how people form first impressions. In a little while you will be playing a game with another person. Because that game won't take very long, and another person in the lab is doing an experiment on personality and problem solving that doesn't take much time either, I was wondering if you would do that experiment first.

All subjects agreed to this request. At this point, each perceiver was given the very same booklet that was used in Experiment 1 . Again, the last puzzle was the priming manipulation in which one of the four groups of priming exemplars was embedded. These were the same exemplars as used in Experiment 1 and were expected to activate the same categories. In this experiment, all subjects listed traits in the same manner as in Experiment 1.

After the perceiver completed the last puzzle, the experimenter thanked her for helping with that study and said,

All right, now we can start the experiment you signed up for. You are going to play a game with another person. In order to know something about that person, we asked an acquaintance of this person to write an account of a recent encounter with him. Please read this account.

At this point, each perceiver was given the same ambiguous description of Donald used in Experiment 1. The perceiver was told to evaluate the 
target person on the basis of her general impression after reading the description. The rating forms, which were the same as those used in Experiment 1, asked the perceiver to assess the target's hostility and, on later pages, other related attributes.

Following completion of the impression measures, a modified prisoners' dilemma game (identical to that used by Jones \& Panitch, 1971) was thoroughly explained to the subjects. Each subject was then tested to be sure that she understood the game. Only when the experimenter was satisfied that both subjects fully understood the game did the game begin. On each of 15 trials, subjects simultaneously expressed their choice on separate slips of paper. After each trial, the slips were collected by the experimenter and each subject's choice and the number of points each subject won were announced. To bolster incentive to play the game, subjects were informed that at the end of the game each point would be converted into 10 cents. After the last trial, each subject was asked to evaluate the other person's hostility and related attributes via the rating form used by the perceiver before the game and by all subjects in the first experiment.

It should be stressed that the targets were, like the perceivers, randomly assigned as partners and were free to behave in any manner they saw fit (within the rules of the game) during the prisoners' dilemma games.

Upon completion of the rating forms, each subject was paid, quizzed for awareness, debriefed, and thanked for her participation. No subject expressed awareness of the experimental hypothesis or connection between the priming manipulation and the rest of the experiment.

\section{Results}

The results of Experiment 2 most relevant to the hypotheses of interest include the initial impressions perceivers formed of the targets (on the basis of priming and reading the alleged description of the target), the game behavior of both targets and perceivers, and the final impressions of targets and perceivers.

Perceivers' judgments of targets. The effect of priming on judgments of the target was demonstrated again with respect to the primary question that asked perceivers to assess the target person's hostility prior to the behavioral interaction. Perceivers primed with exemplars of extreme hostility or moderate nonhostility evaluated the target person as being less hostile than did perceivers primed with exemplars of extreme nonhostility or moderate hostility, $F(1,36)=18.26 p<.001$. Means for this analysis and results of tests of simple effects can be seen in Table 3. In addition to this interaction was a main effect for extremity of prime, $F(1,36)=4.57, p<.05$, in which, overall, priming with moderate exemplars led to higher ratings of hostility than did priming with extreme exemplars. This is, of course, qualified by the interaction of category and extremity reported earlier. This is, in fact, a replication of one part of the extremity by trait listing interaction found in Experiment 1, because in this experiment all perceivers listed traits.

Supplementary impression measures. Analyses of the remainder of the questionnaire given to perceivers immediately following priming and the description of the target revealed an unexpected finding that differed from the first experiment. As in Experiment 1, composites of positive items, negative items, and all items on the questionnaire were made by summing across each item, respectively. Again, Cronbach's alphas for these scales were relatively high (negative scale $=.83$, positive $=$ .85 , total $=.79$ ), so 2 (category primed) $\times 2$ (extremity of category primed) ANOVAs were conducted on each of the composite
Table 3

Dependent Measures for Experiment 2

\begin{tabular}{|c|c|c|c|c|}
\hline \multirow[b]{3}{*}{ Measure } & \multicolumn{4}{|c|}{ Prime } \\
\hline & \multicolumn{2}{|c|}{ Moderate } & \multicolumn{2}{|c|}{ Extreme } \\
\hline & Nonhostile & Hostile & Nonhostile & Hostile \\
\hline $\begin{array}{l}\text { Mean evaluation of } \\
\text { target's hostility } \\
\text { Supplementary ratings }\end{array}$ & $5.00_{\mathrm{a}}$ & $6.70_{b}$ & $6.10_{\mathrm{ab}}$ & $3.40_{\mathrm{c}}$ \\
\hline $\begin{array}{l}\text { Target's friendliness } \\
\text { Target's kindness } \\
\text { Would like to know }\end{array}$ & $\begin{array}{l}4.30 \mathrm{a} \\
3.80_{\mathrm{a}} \\
3.40_{\mathrm{ab}}\end{array}$ & $\begin{array}{l}5.20_{\mathrm{a}} \\
5.30_{\mathrm{a}} \\
5.11_{\mathrm{a}}\end{array}$ & $\begin{array}{l}5.20_{\mathrm{a}} \\
5.10_{\mathrm{a}} \\
4.60_{\mathrm{ab}}\end{array}$ & $\begin{array}{l}3.90_{\mathrm{a}} \\
4.10_{\mathrm{ab}} \\
2.90_{\mathrm{b}}\end{array}$ \\
\hline $\begin{array}{l}\text { Mean number of } \\
\text { competitive } \\
\text { choices }\end{array}$ & & & & \\
\hline $\begin{array}{l}\text { Perceiver } \\
\text { Target }\end{array}$ & $\begin{array}{l}8.4_{\mathrm{a}} \\
9.1_{\mathrm{b}}\end{array}$ & $\begin{array}{l}9.2_{\mathrm{b}} \\
8.4_{\mathrm{a}}\end{array}$ & $\begin{array}{l}9.0_{b} \\
8.3_{\mathrm{a}}\end{array}$ & $\begin{array}{l}8.3_{\mathrm{a}} \\
9.7_{\mathrm{b}}\end{array}$ \\
\hline $\begin{array}{l}\text { Mean number of points } \\
\text { won }\end{array}$ & & & & \\
\hline Perceiver & $7.9_{\mathrm{a}}$ & $10.4_{b}$ & $9.6_{\mathrm{b}}$ & $7.5_{\mathrm{a}}$ \\
\hline Target & $9.3_{b}^{\circ}$ & $7.8_{a}^{\circ}$ & $8.5_{\mathrm{a}}$ & $9.5_{\mathrm{b}}$ \\
\hline $\begin{array}{c}\text { Mean ratings by targets } \\
\text { of perceivers' }\end{array}$ & & & & \\
\hline $\begin{array}{l}\text { hostility } \\
\text { Mean final impressions }\end{array}$ & $3.10 \mathrm{a}$ & $5.80_{\mathrm{b}}$ & $5.50_{\mathrm{bc}}$ & $3.80_{\mathrm{ac}}$ \\
\hline Target's friendliness & $4.10_{\mathrm{m}}$ & $5.70_{b}$ & $5.60_{b}$ & $4.30_{\mathrm{a}}$ \\
\hline $\begin{array}{l}\text { Target's kindness } \\
\text { Target's }\end{array}$ & $3.90 \mathrm{a}$ & $5.70_{b}$ & $5.70_{b}$ & $4.40_{\mathrm{a}}$ \\
\hline competitiveness & $8.00_{\mathrm{a}}$ & $7.10_{\mathrm{ab}}$ & $6.00_{b}$ & $7.90 \mathrm{a}$ \\
\hline Target's bitterness & $5.30 \mathrm{n}$ & $4.40_{\mathrm{ab}}$ & $3.20_{b}$ & $4.90_{\mathrm{ab}}$ \\
\hline Would work with & $4.00 \mathrm{a}$ & $5.90_{b c}$ & $6.40_{c}$ & $4.40_{\mathrm{ab}}$ \\
\hline Would like to know & $4.33 \mathrm{~g}$ & $6.22_{\mathrm{a}}$ & $6.00_{\mathrm{a}}$ & $4.50_{\mathrm{a}}$ \\
\hline $\begin{array}{l}\text { Behavior reflects } \\
\text { internal causes }\end{array}$ & $5.44_{\mathrm{ab}}$ & $4.56_{b}$ & $4.75_{\mathrm{b}}$ & $6.75_{\mathrm{a}}$ \\
\hline
\end{tabular}

Note. Higher scores indicate that more of the perceived characteristic is attributed to the individual. Cell means in the same row not sharing a common subscript differ beyond $p=.05$, as revealed by relevant test of simple effects (except for mean number of competitive choices, where $p=.07$ ). For measures in which means for both perceiver and target are presented simultaneously, any cell mean not sharing a common subscript differs by relevant test of simple effects.

scores. The only analysis to reveal an interaction between category primed and extremity was that conducted on the positive items, $F(1,36)=6.81, p=.01$. There were no main effects. This interaction, however, did not take the predicted form. For three of the items on this scale taken by themselves, this same interaction also obtained. These were questions assessing how much the perceiver would like to know the target, how friendly, and how kind the perceiver thought the target was, $F(1,35)=$ $4.89, p=.03, F(1,36)=4.26, p=.05, F(1,36)=5.54, p=$ .02 , respectively. The surprising finding here is that these ratings are in the opposite direction from the ratings of the target's hostility, which all perceivers answered first. That is, subjects

\footnotetext{
${ }^{2}$ For a cooperative choice, the player's expected outcome was .5 points and her partner's was 1.5 . For a competitive choice, the player's expected outcome was 1.0 and her partner's was 0 . Actual outcomes ranged from joint competition yielding 0 points to each player, cooperation by $\mathrm{A}$ and competition by $\mathrm{B}$ giving $\mathrm{A} 0$ points and $\mathrm{B} 2$ points, to joint cooperation giving each player 1 point.
} 
primed with exemplars of extreme hostility or moderate nonhostility rated the target as less friendly and kind, and they wanted to know the target less than did subjects primed with exemplars of extreme nonhostility or moderate hostility.

An explanation of these surprising data may come from the social interaction literature. Darley and Berscheid (1967) have demonstrated that when people anticipate future interaction with another person, they tend to enhance their global opinion of that person. Given that priming had affected their rating of the target in the first measure, the perceivers may have used the remainder of the questionnaire to give a rosier view of the target. (This did not occur in Experiment 1 because subjects did not anticipate future interaction with the person they evaluated.) Baumeister and Jones (1978) have demonstrated that when a negative characteristic about a person is known to another, the labeled person will attempt to compensate by exaggerating other favorable independent characteristics. Something akin to this may have occurred with perceivers, who, having rated the target's hostility, were then faced with evaluating the target along other dimensions.

Whatever the source of these anomalous data, there are several reasons for discounting their importance. First, the data on the assessment of the target's hostility, taken prior to the collection of these data, were the main focus of interest with respect to the experimental hypotheses and were identical to the data collected in Experiment 1. Second, these anomalous effects did not obtain in Experiment 1, and they were likely to be due to the anticipation of future interaction with the target rather than to an expression of privately held beliefs. Finally, the behavioral data to be presented were consistent with the ratings of hostility and not at all consistent with the data collected on the supplementary measures.

Behavioral effects: Playing the game. The number of competitive choices subjects made was analyzed by means of a 2 (extremity: moderate vs. extreme priming exemplars) $\times 2$ (category primed: nonhostile vs. hostile) $\times 2$ (dyad member: perceiver vs. target) $\times 2$ (block: first vs. second half of trials in the prisoners' dilemma) ANOVA with dyad and block within factors. The impact of priming on behavior can be seen in the significant three-way interaction of extremity, category primed, and member of dyad, $F(1,36)=6.62, p=.01$. It was expected that perceivers who expected nonhostile targets (those primed with exemplars of extreme hostility or moderate nonhostility) would compete less than would perceivers who expected hostile partners (i.e., those primed with exemplars of moderate hostility or extreme nonhostility). Simple effects of this interaction revealed that not only was this the case, but perceivers tended to gauge their level of competition with that of their partner, such that perceivers who expected nonhostile partners competed slightly less than did targets with whom they were paired, whereas perceivers who expected hostile partners competed slightly more than did their targets, $t(36)=1.82, p=.07$. The means for this interaction are presented in Table 3 .

In addition to this three-way interaction, there was a significant main effect for block, $F(1,36)=21.12, p<.001$, in which all subjects competed more in the second block of seven trials than in the first block. There was also an extremity by block interaction, $F(1,36)=3.95, p=.05$, in which the increase in competition across blocks tended to be greater for dyads in which perceivers had been exposed to moderate primes than for dyads in which perceivers had been exposed to extreme exemplars. Finally, there was a significant three-way interaction of category, dyad, and block, $F(1,36)=4.31, p=.04$, in which perceivers exposed to exemplars of nonhostility and targets paired with perceivers exposed to exemplars of hostility tended to increase their competition more, although both perceivers and targets competed more in the second block. No other effect approached significance.

These differences in winnings were manifested in differences in actual points won at the end of the game, as revealed by a 2 (category primed: hostile vs. nonhostile) $\times 2$ (extremity of category primed: moderate vs. extreme) $\times 2$ (dyad member: perceiver vs. target) ANOVA, with the last factor within subjects. This analysis revealed a significant three-way interaction, $F(1$, $36)=6.47, p=.01$, with no other effects approaching significance. Tests of simple effects revealed that perceivers primed with exemplars of extreme nonhostility or moderate hostility won significantly more points than did their target/partners, in addition to having won more than those perceivers who had been primed with exemplars of extreme hostility or moderate nonhostility, $t(36)=2.54, p<.01$.

Final impressions. Following the interaction, both perceiver and target were given the same questionnaire to complete. In addition to the 11 questions asked the perceiver before the game was played, there were also questions designed to determine whether subjects attributed their own and their partner's behavior to situational or dispositional causes.

Perhaps the strongest evidence that priming with different exemplars led to different behavior on the part of the perceivers was found in an analysis of the targets' impressions of the perceivers' hostility following the interaction. Because initially there were no differences between targets' assessments of perceivers' hostility (by virtue of their random assignment to condition), their final impressions were based solely on the behavior of the perceivers during the course of the interaction, and any differences in impressions reflect the differences in behavior displayed by perceivers during the course of the game. Following the game, when targets assessed the perceivers' hostility on the same 11-point scale used throughout the experiment, differences indeed existed. A 2 (extremity of priming exemplars) $\times$ 2 (category primed) ANOVA revealed a significant interaction, $F(1,36)=9.87, p=.003$, in which targets whose partners had been primed with exemplars of extreme nonhostility or moderate hostility evaluated those perceivers as being more hostile than did targets whose partners had been primed with exemplars of extreme hostility or moderate nonhostility. Both the assimilation and contrast effects produced in judgments of targets' hostility were reciprocated in judgments of those perceivers. Neither main effect approached significance.

These results are really quite interesting when compared with the findings of Jones and Panitch (1971). In their study, the perceivers were told before the game that their partner did not like them. As a consequence of the perceivers' behavior during the game, the targets, in fact, came to dislike them. In the experiment presented here, the perceiver came to expect a hostile or nonhostile target through priming, and the target then reciprocated that evaluation, again as a consequence of the perceiver's behavior. 
The same analysis was conducted on perceivers' postinteraction evaluations of targets. Following the interaction, there were no differences across conditions in perceptions of the targets' hostility, $F(1,36)=1.44$, ns. However, seven of the supplementary measures attained statistically significant interactions. These were perceptions of the targets' competitiveness, $F(1$, $36)=4.98, p<.05$; bitterness, $F(1,36)=3.98, p=.05$; desire to work with, $F(1,36)=8.68, p<.01$; and desire to know, $F(1$, $30)=4.63, p<.05$. In all of these interactions, perceivers who had originally evaluated their partner as being relatively nonhostile now evaluated them more negatively than did perceivers who had initially expected a relatively more hostile partner. Perceivers who expected their partners to be nonhostile also came to believe that their partners were less kind and less friendly than did perceivers who expected a hostile partner, $F(1,36)=$ $11.78, p<.01$, and, $F(1,36)=10.30, p<.01$, respectively. Perceivers expecting to interact with a relatively nonhostile target also believed more strongly that their partners' behavior reflected underlying personality characteristics than did those perceivers who expected a hostile target, $F(1,30)=4.84, p<$ .05. No other effects obtained.

Given the anomalies in the supplementary measures prior to the behavioral interaction, interpretation of these postinteraction measures is not entirely straightforward. They in fact tend to show the same between-condition differences exhibited in the preinteraction supplementary measures, whereas the perceivers' evaluations of the targets' hostility have changed. Whether these changes were influenced by the targets' behavior or were merely assimilations to the earlier supplementary judgments is not clear. Given the aforementioned behavioral effects, the former explanation seems more likely. It is possible, though, that whatever the cause of the anomalous preinteraction ratings, those ratings remained stable throughout the course of the interaction and don't represent a now true reflection of the perceivers' beliefs about the target. That they are being presented as evidence that perceivers changed their beliefs has to do with the intuitive plausibility of the ratings at the time they were collected. The preinteraction ratings are anomalous owing to their inconsistency with the hostility ratings, which had just been made and which were consistent with the earlier published findings of Herr et al. (1983). If one assumes that those initial hostility ratings are in fact valid, as must be done to parsimoniously handle the behavioral data, that the postinteraction evaluations described above represent true perceiver beliefs makes some sense. The person expected to be hostile turned out not to be hostile and, as a consequence, was evaluated as being more kind and so forth than a person who initially was expected to be nonhostile and who seemingly took advantage of the perceiver.

\section{General Discussion}

The present experiments demonstrate that unobtrusive exposure to exemplars of social categories has profound consequences for the perception of another person and subsequent behavioral interaction with that person.

The first experiment conceptually replicated and extended the findings of Herr et al. (1983) by demonstrating that subjects primed with exemplars of moderate categories evaluated an ambiguously described target person consistently with those categories, whereas subjects exposed to exemplars of extreme categories evaluated the same ambiguously described target person in the opposite direction from the activated category. This experiment demonstrated for the first time that, following priming of a social category, both assimilation and contrast effects of judgment occur. Subjects primed with exemplars of moderate hostility or extreme nonhostility evaluated the target person as being more hostile than did persons primed with exemplars of extreme hostility or moderate nonhostility. Although Higgins et al. (1977) and Srull and Wyer (1978) both primed social categories, neither examined the possibility of contrast effects following priming. As seen here, such contrast effects do occur under certain prespecified conditions.

Experiment 2 again demonstrated the judgment effects found in the first experiment. In addition, however, when subjects interacted with the person whose hostility they evaluated, they behaved consistently with that evaluation, which resulted in their partner's coming to evaluate them as they had first evaluated their partner. Hence, the recent activation of a social category (hostility) by unobtrusive exposure to exemplars of that category not only can have effects on the judgment or categorization of an ambiguously described target person, but it can also affect behavior directed toward that target person.

The expectancy instilled by priming was not so strong, however, as to be irrefutable by the behavior of the target. The perceiver seemed, rather, to gauge her level of competition to that of the target, such that, if the target was expected to be hostile, the perceiver competed slightly more than the target, whereas if the target was expected to be nonhostile, the perceiver competed slightly less than the target. By the end of the game, though, the perceiver's initial impression had become more consistent with the behavior of the target. These results might interestingly be compared with the findings of Swann and Ely (1984), who also demonstrated that initial impressions held by perceivers may change during an interaction with a target.

As for the targets, they came to reciprocate the hostility impressions originally held by the perceivers with whom they were matched. Targets paired with perceivers who had been primed with exemplars of moderate hostility or extreme nonhostility came to evaluate the perceiver as being more hostile than did targets who were paired with perceivers who had been primed with exemplars of extreme hostility or moderate nonhostility. That is, the priming-induced contrast and assimilation effects of judgments were reciprocated by the person who had been judged first. What is unique about these findings is the manner in which the perceivers' initial impression of the target was formed. Unlike earlier reports of reciprocation of impression and self-fulfilling prophecy (e.g., Jones \& Panitch, 1971; Snyder \& Swann, 1978), in this experiment the perceiver was never told that the target was or was not hostile, or did or did not like her. All perceivers read the very same information about the target. Also, all targets (at least initially) displayed the same degree of competition. The determinant of the perceiver's impression of the target, her behavior toward the target, and, in turn, the target's behavior toward and impression of the perceiver was the category activated by unobtrusive exposure to one of the four lists of exemplars.

Although there were unexpected and contradictory findings with the supplementary measures in Experiment 2, there is lit- 
the question that both hostility and nonhostility were in fact primed and had profound effects on behavior. Again, kind or friendly or some semantically related category may have been activated rather than, or in addition to, hostility. For such to occur, however, the hostility ratings would have to be considered anomalous, and a rather different, less parsimonious interpretation of the behavioral data considered. In fact, the rating effects for hostility (the first judgment subjects made) were strong, replicated precisely the findings in Experiment 1, and appeared entirely consistent with the hypothesized effects of having primed hostility. Clearly, when the perceivers evaluated the target's hostility, they did so in the very manner found in Herr et al. (1983) and in Experiment 1. No model of priming would predict such inconsistent ratings by an individual when a single target is being evaluated. The sole difference methodologically between Experiment 1 and Experiment 2 rested in the perceiver's knowledge, at the time of first evaluating the target, that she would interact with that target in the near future. Given the past research implicating judgments of hostility as influential in predicting level of competition (Jones \& Panitch, 1971; Kelley \& Stahelski, 1970) and the lengths to which we must go to make the supplementary ratings consistent with behavior (perceivers expecting unfriendly targets fatalistically competed less so as not to arouse them and make them compete more, whereas those perceivers expecting friendly partners took advantage of their partners and competed more?), it seems reasonable to assume that hostility was primed and that it mediated behavior in the social interaction.

It is clear from this study, then, that the most parsimonious explanation of the behavioral data is the perceivers' priminginduced evaluations of targets' hostility. Perhaps the strongest evidence that the hostility ratings are real and the supplementary measures artifactual rests in the targets' evaluations of the perceivers' hostility, evaluations that mirror the perceivers' original ratings of the targets' hostility. The sole influence on these ratings was the behavior of the perceiver, behavior inconsistent with the friendly and kind ratings and consistent with the hostility ratings.

A self-fulfilling prophecy, whereby targets reciprocate the perceivers' behavior, did not occur. The chain of events necessary for the self-fulfilling prophecy (see Darley \& Fazio, 1980) could have been broken at any link. Clearly though, perceivers held different expectancies of the targets' behavior. Equally clearly, these differences were translated into different behavior directed toward the target. Possibly, were the setting not a game and defection not rewarded by perceivers expecting nonhostile partners, the self-fulfilling prophecy might have obtained.

This demonstrated link between the activation of a category and behavior consistent with that category serves to strengthen the case of the social cognition perspective (cf. Carroll \& Payne, 1976; Manis, 1977) whereby information processing is seen as a mediator of behavior. After the category has been activated and the judgment of the target person made, the expectancy determines the behavior of the perceiver. Conceivably, the process is similar to the way attitudes are posited to guide behavior (cf. Fazio, Powell, \& Herr, 1983). That is, the activated category may serve as a filter through which ongoing events are screened. Ambiguous and/or expectancy congruent events may be perceived consistently with the activated category. That is, the per- ceiver should conclude that these events are consistent with her expectancy and reciprocate. In Experiment 2, the cooperation on the part of the target may be viewed as ambiguous and subject to interpretational differences caused by the activated category. The data are consistent with the notion that subjects interpreted cooperation differently, depending on which category had been primed. Those perceivers for whom the category hostile had been primed interpreted cooperation as a strategic ploy on the part of the target to induce them to cooperate, rather than as an indication of an underlying disposition of cooperativeness. Perceivers for whom the category nonhostile had been primed interpreted the same cooperative behavior as an indication that their partner was, in fact, cooperative at heart. Unambiguous and/or expectancy incongruent behavior should not be filtered through the category. Instead, perceivers should recognize that the target is not acting as expected and should either change their own behavior toward the target, change their evaluation of the target, or both. That the perceivers who expected a nonhostile partner did not change their behavior and compete more may have reflected their belief that the target's cooperative choices would be forthcoming. That belief, of course, was shattered when the game concluded and perceivers were asked to evaluate the target. These notions were not completely tested in this study, and further research might examine the consequences of behavior inconsistent with the priming-induced expectancy on the behavior of the perceiver.

A second area of further research might well address the question of whether the behavioral effects would obtain if an overt judgment of the target's hostility had not been made. That is, would perceivers spontaneously evaluate the targets following priming? Earlier work by Sherman, Ahlm, Berman, and Lynn (1978) suggests that only when an overt judgment about an object is made will the context in fact influence behavior. More recently, however, Winter, Uleman, and Cunniff (1985) have suggested that social judgments are in fact made automatically. To the extent that social judgments are automatically made, these findings are all the more important for illuminating priming's influence on behavior.

\section{References}

Bargh, J. A., \& Pietromonaco, P. (1982). Automatic information processing and social perception: The influence of trait information presented outside of conscious awareness on impression formation. Journal of Personality and Social Psychology, 43, 437-449.

Baumeister, R. F., \& Jones, E. E. (1978). When self-presentation is constrained by the target's knowledge: Consistency and compensation. Journal of Personality and Social Psychology, 36, 608-618.

Carroll, J. S., \& Payne, J. W. (Eds.). (1976). Cognition and social behavior: Hillsdale, NJ: Erlbaum.

Darley, J. M., \& Berscheid, E. (1967). Increased liking as a result of the anticipation of personal contact. Human Relations, 20, 29-39.

Darley, J. M., \& Fazio, R. H. (1980). The origin of self-fulfilling prophecies in a social interaction sequence. American Psychologist, 35, 867881.

Fazio, R. H., Powell, M. C., \& Herr, P. M. (1983). Toward a process model of the attitude-behavior relation: Accessing one's attitude upon mere observation of the attitude object. Journal of Personality and Social Psychology, 44, 723-735.

Herr, P. M., Sherman, S. J., \& Fazio, R. H. (1983). On the consequences 
of priming: Assimilation and contrast effects. Journal of Experimental Social Psychology, 19, 323-340.

Higgins, E. T., Bargh, J. A., \& Lombardi, W. (1985). Nature of priming effects on categorization. Journal of Experimental Psychology: Learning, Memory, and Cognition, 11, 58-69.

Higgins, E. T., \& King, G. A. (1981). Accessibility of social constructs: Information-processing consequences of individual and contextual variability. In N. Cantor \& J. F. Kihlstrom (Eds.), Personality, cognition, and social interaction (pp. 69-121). Hillsdale, NJ: Erlbaum.

Higgins, E. T., King, G. A., \& Mavin, G. H. (1982). Individual construct accessibility and subjective impressions and recall. Journal of Personality and Social Psychology, 43, 35-47.

Higgins, E. T., Rholes, W. S., \& Jones, C. R. (1977). Category accessibility and impression formation. Journal of Experimental Social Psychology, 13, 141-154.

Jones, S. C., \& Panitch, D. (1971). The self-fulfilling prophecy and interpersonal attraction. Journal of Experimental Social Psychology, 7, 356-366.

Kelley, H. H., \& Stahelski, A. J. (1970). Social interaction and competitors' and cooperators' beliefs about others. Journal of Personality and Social Psychology, 16, 66-91.

Manis, M. (1977). Cognitive social psychology. Personality and Social Psychology Bulletin, 3, 550-566.

Martin, L. L. (1986). Set/reset: The use and disuse of concepts in impression formation. Journal of Personality and Social Psychology, 51 , 493-504.

Ostrom, T. M., \& Upshaw, H. S. (1968). Psychological perspective and attitude change. In A. G. Greenwald, T. C. Brock, \& T. M. Ostrom (Eds.), Psychological foundations of attitudes (pp. 217-242). New York: Academic Press.

Sherman, S. J., Ahlm, K., Berman, L., \& Lynn, S. (1978). Contrast effects and their relationship to subsequent behavior. Journal of Experimental Social Psychology, 14, 340-350.

Snyder, M., \& Swann, W. B. (1978). Behavioral confirmation in social interaction: From social perception to social reality. Journal of Experimental Social Psychology, 14, 148-162.

Snyder, M., Tanke, E. D., \& Berscheid, E. (1977). Social perception and interpersonal behavior: On the self-fulfilling nature of social stereotypes. Journal of Personality and Social Psychology, 35, 656-666.

Srull, T. K. \& Wyer, R. S. (1978). Category accessibility and social perception: Some implications for the study of person memory and interpersonal judgments. Journal of Personality and Social Psychology, 37 , 841-856.

Srull, T. K., \& Wyer, R. S. (1979). The role of category accessibility in the interpretation of information about persons: Some determinants and implications. Journal of Personality and Social Psychology, 38. 1660-1672.

Swann, W. B., \& Ely, R. J. (1984). A battle of wills: Self-verification versus behavioral confirmation. Journal of Personality and Social Psychology, 46, 1287-1302.

Upshaw, H. S. (1962). Own attitude as an anchor in equal appearing intervals. Journal of Abnormal and Social Psychology, 64, 85-96.

Winter, L., Uleman, J. S., \& Cunniff, C. (1985). How automatic are social judgments? Journal of Personality and Social Psychology, 49, 904-917.

Word, C. O., Zanna, M. P., \& Cooper, J. (1974). The nonverbal mediation of self-fulfilling prophecies in interracial interaction. Journal of Experimental Social Psychology, 10, 109-120.

Wyer, R. S., \& Srull, T. K. (1980a). Category accessibility: Some theoretical and empirical issues concerning the processing of social stimulus information. In E. T. Higgins, C. P. Herman, \& M. P. Zanna (Eds.), Social cognition: The Ontario Symposium. Hillsdale, NJ: Erlbaum.

Wyer, R. S., \& Srull, T. K. (1980b). The processing of social stimulus information: A conceptual integration. In R. Hastie, T. Ostrom, E. Ebbeson, R. Wyer, D. Hamilton, \& D. Carlston (Eds.), Cognitive bases of impression formation and person memory. Hillsdale, NJ: Erlbaum.

Received December 7, 1984

Revision received May 23, 1986 